\title{
Diabetes and kidney function
}

\section{Editorial}

Diabetes Mellitus, commonly known as diabetes and its associated diseases are increasing at an alarming rate and causing significant health threat to humans worldwide. Diabetes exists in two forms, type 1 and type 2 . In type I diabetes, the body cannot make enough insulin to metabolize glucose, whereas in type II diabetes body loses the ability to effectively utilize insulin. While the type 1 diabetes develops early in the childhood (thus termed as juvenile diabetes) the type 2 diabetes occurs commonly in people over 40 (thus termed as adult diabetes). Although both forms of diabetes can be controlled through the infusion of insulin, unlike type 1 diabetes that requires insulin shots for life, type 2 diabetes can be controlled through physical exercise, diet and medication. Some communities are particularly vulnerable to type 2 diabetes and include Asian Americans, African Americans, Latin Americans and American Indians. ${ }^{1,2}$

The diabetic patients are highly prone to developing kidney disease, especially when appropriate measures are not taken to control diabetic symptoms (Figure 1). The nephropathy associated with type 2 diabetes is commonly known as Diabetic Nephropathy (DN) and is the leading cause of End Stage Renal Disease (ESRD). In fact, $30 \%$ of patients with type 1 diabetes and $10-40 \%$ patients with type 2 diabetes may eventually lose their kidney function and end up in the need of either dialysis or renal transplant. ${ }^{2}$ One of the major complications of diabetes is injury to blood vessels of kidney that prevents kidney from functioning normally. This results in fluid and salt retention leading to increase in weight and morbidity. One of the primary symptoms of the onset of kidney disease in a diabetic patient is increased excretion of protein in the urine. This may be accompanied by weight gain, swelling of ankles, increased frequency of urination during night time and elevated blood pressure. If the diabetes is not controlled, a progressive loss of kidney function occurs leading to ESRD. The only treatments left in such cases are a kidney transplant, hemodialysis or peritoneal dialysis. All these measures may require significant lifestyle changes and do not guarantee a successful outcome. Thus better awareness of this disease and prevention through a controlled diet and regular checkups are two strong measures that may help reduce the incidence of kidney disease in a diabetic patient.

Despite the best efforts through standard care and therapeutic interventions, renal complications are only modestly reduced in diabetic patients, indicating an urgent need for early identification and treatment of these complications. ${ }^{1,3}$ Global efforts are underway to identify novel biomarkers that can predict the early onset of kidney damage and find new therapies and/or drugs that will arrest the development of ESRD observed in DKD patients. Vast amount of research in this area suggests that DKD is also associated with other complications such as cardiovascular (CV) and therefore a multipronged approach may be required to target the treatment of $\mathrm{DKD}^{3-7}$ Some of the targeted therapies that are commonly used but have limited scope in controlling DKD are glycemic control, antihypertensive and dyslipidemia improvement. ${ }^{3}$ Data from clinical trials "Diabetes Control and Complications" and "United Kingdom Prospective Diabetes" suggested that adequate control of hyperglycemia significantly decreases $(33-84 \%)$ the progression of macroalbuminuria from microalbuminuria. ${ }^{8}$ Among the drugs that

Volume I Issue 2 - 2014

\section{Deepak Nihalani \\ Renal Electrolyte and Hypertension Division, University of Pennsylvania, USA}

Correspondence: Deepak Nihalani, Renal Electrolyte and Hypertension Division, University of Pennsylvania, 405D CRB, 415 Curie Blvd, Philadelphia, PA 19104, USA, Tel 2I 58980192, Fax 215 5736264,Email deepakn@mail.med.upenn.edu

Received: May 01, 2014 | Published: May 02, 2014

target hyperglycemia, metformin and sulphonylureas are commonly prescribed; however, they have limited therapeutic applicability and are less preferred over insulin therapy. ${ }^{3,6}$ There is ample evidence to support the use of antihypertensive drugs such as ACE (angiotensin converting enzyme) inhibitors and Angiotensin Receptor (AR) blockers that target the Renin Angiotensin Aldosterone System (RAAS) in benefiting DKD patients. ${ }^{3,9}$ Diabetic patients may require different combinations of antihypertensive drugs in controlling the disease and therefore, may offer limited benefits to DKD patients. Statins are frequently used in DKD patients for lowering LDL cholesterol and have been shown effective in reducing CV complications. However, trials conducted to determine the protective effect of statins on proteinuria and progression of CKD, has yielded mixed results and therefore, statin therapy is not recommended for renal patients. ${ }^{3,10-14}$

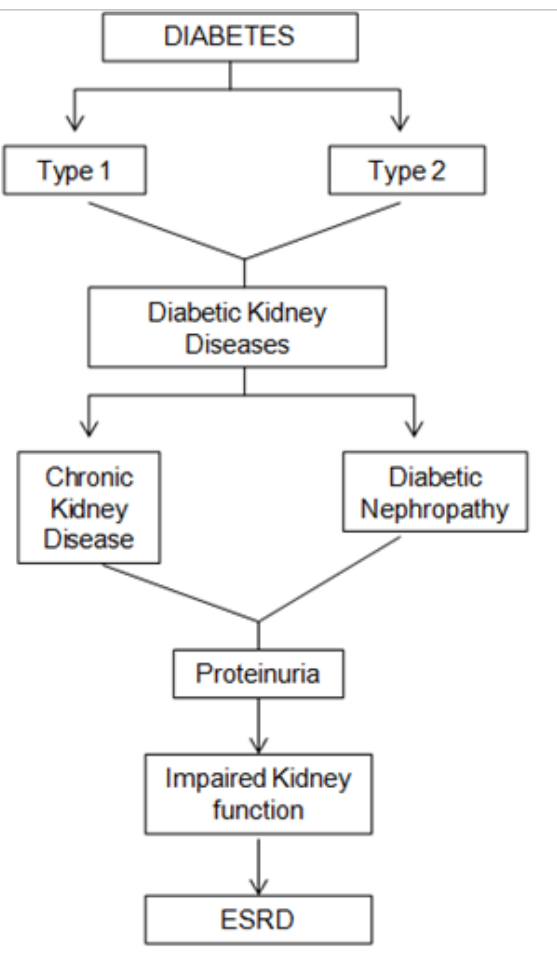

Figure I Diabetes and kidney diseases. 
With a continuous increase in DKDs worldwide, there is urgent need to identify novel therapeutic targets including proteins and molecular mechanisms that mediate the pathogenesis of DKD. A significant step forward in this direction had been provided by an intense research in the field of podocytes that are major components of the glomerular filtration assembly whose dysfunction is strongly associated with the progression of ESRD. Various animal and human studies have shown loss of podocytes under diabetic nephropathic conditions. More importantly, studies have shown that therapies that preserve podocyte function have remarkable effect on restoring renal function and prevent proteinuria. ${ }^{15,16}$ Thus cellular mechanisms that regulate podocyte structure and function such as autophagy have become prime therapeutic targets to combat DKD. ${ }^{17}$ Additionally, various endocrine and metabolic pathways such as renin angiotensin system and insulin receptor pathway have been shown to play a key role in regulating podocyte function. ${ }^{16}$ As opposed to the conventional therapies to treat DKDs that target multiple cellular sites, podocytes present a specific target that has significant potential to preserve renal function and offer reduced side effects. However, research in this field is in its infancy and will require extensive worldwide effort to convert these concepts from benchside research to bedside research of clinical significance. With a steady increase in funding for diabetes research, there is a great deal of optimism that a cure for diabetes is in future. However, the pathogenicity of diabetes is highly complex and no single therapy may be sufficient to combat this disease. Thus we need to explore novel avenues of research that are directed towards identifying novel molecules and pathways that can be therapeutically targeted for improving the lives of DKD patients.

\section{Acknowledgements}

None.

\section{Conflict of interest}

Author declares that there is no conflict of interest.

\section{References}

1. Fernandez Fernandez B, Elewa U, et al. 2012 update on diabetic kidney disease: the expanding spectrum, novel pathogenic insights and recent clinical trials. Minerva Med. 2012;103(4):219-234.

2. http://www.usrds.org

3. Gosmanov AR, Wall BM, Gosmanova EO. Diagnosis and treatment of diabetic kidney disease. Am J Med Sci. 2014;347(5):406-413.
4. Jim B, Santos J, Spath F, et al. Biomarkers of diabetic nephropathy, the present and the future. Curr Diabetes. 2012;8(5):317-328.

5. Kolset SO, Reinholt FP, Jenssen T. Diabetic nephropathy and extracellular matrix. J Histochem Cytochem. 2012;60(12):976-986.

6. Liang S, Li Q, Zhu HY, et al. Clinical Factors Associated With the Diagnosis and Progression of Diabetic Nephropathy. Cell Biochem Biophys. 2104.

7. Tonolo G, Cherchi S. Tubulointerstitial disease in diabetic nephropathy. Int J Nephrol Renovasc Dis. 2014;7:107-115.

8. Predictors of the development of microalbuminuria in patients with Type 1 diabetes mellitus: a seven-year prospective study. The Microalbuminuria Collaborative Study Group. Diabet Med. 1999;16(11):918-925.

9. Lewis G, Maxwell AP. Risk factor control is key in diabetic nephropathy. Practitioner. 2014;258(1768):13-17.

10. Atthobari J, Brantsma AH, Gansevoort RT, et al. The effect of statins on urinary albumin excretion and glomerular filtration rate: results from both a randomized clinical trial and an observational cohort study. Nephrol Dial Transplant. 2006;21(11):3106-3114.

11. Douglas K, O’Malley PG, Jackson JL. Meta-analysis: the effect of statins on albuminuria. Ann Intern Med. 2006;145(2):117-124.

12. Lee TM, Lin MS, Tsai CH, et al. Add-on and withdrawal effect of pravastatin on proteinuria in hypertensive patients treated with AT receptor blockers. Kidney Int. 2005;68(2):779-787.

13. Ozsoy RC, Koopman MG, Kastelein JJ, et al. The acute effect of atorvastatin on proteinuria in patients with chronic glomerulonephritis. Clin Nephrol. 2005;63(4):245-249.

14. Ruggenenti P, Perna A, Tonelli M, et al. Effects of add-on fluvastatin therapy in patients with chronic proteinuric nephropathy on dual reninangiotensin system blockade: the ESPLANADE trial. Clin J Am Soc Nephrol. 2010;5(11):1928-1938.

15. De Cosmo S, Menzaghi C, Prudente S, et al. Role of insulin resistance in kidney dysfunction: insights into the mechanism and epidemiological evidence. Nephrol Dial Transplant. 2013;28(1):29-36.

16. Diez-Sampedro A, Lenz O, Fornoni A. Podocytopathy in diabetes: a metabolic and endocrine disorder. Am J Kidney Dis. 2011;58(4):637-646.

17. Yamahara K, Yasuda M, Kume S, et al. The role of autophagy in the pathogenesis of diabetic nephropathy. J Diabetes Res. 2013:193757. 